\title{
COMPOSIÇÃO MINERAL DO PRODUTO COMERCIAL DA ERVA-MATE (Ilex paraguariensis St. Hil.)
}

\author{
MINERAL COMPOSITION OF A COMMERCIAL PRODUCT FROM MATE-HERB \\ (Ilex paraguariensis St. Hil.)
}

\section{Reges Heinrichs ${ }^{1}$ Eurípedes Malavolta ${ }^{2}$}

\section{RESUMO}

Com o objetivo de quantificar e avaliar a concentração mineral na matéria seca e na infusão de erva-mate tipo chimarrão, selecionaram-se três amostras comerciais, com ampla aceitação pelo consumidor, as quais foram amostradas com quatro repetições. Na industrialização da erva-mate tipo chimarrão, são utilizadas folhas, pecíolos e ramos finos, tendo uma composição aproximada de $30 \%$ ramos e $70 \%$ folhas, que são beneficiados para posterior comercialização. $O$ preparo das amostras e as análises foram efetuadas no Laboratório de Nutrição Mineral de Plantas do Centro de Energia Nuclear na Agricultura (CENA/USP). Para determinação da composição mineral da matéria seca, as amostras foram moídas em moinho "tipo Willey”, com posterior digestão e determinação dos teores de $N$, $\mathrm{P}, \mathrm{K}, \mathrm{Ca}, \mathrm{Mg}, \mathrm{S}, \mathrm{B}, \mathrm{Cu}, \mathrm{Fe}, \mathrm{Mn}, \mathrm{Ni}, \mathrm{Zn}, \mathrm{Al}, \mathrm{Cd}, \mathrm{Co}, \mathrm{Cr}, \mathrm{Na}$ e Pb. Para determinação dos elementos na infusão, utilizaram-se $70 \mathrm{~g}$ de erva-mate para um litro de água desionizada a $80^{\circ} \mathrm{C}$, simulando-se a temperatura para chimarrão, mantendo-se até esfriar $e$ posteriormente coando-se. Os elementos analisados na infusão foram os determinados na matéria seca e, além destes, $\mathrm{Mo}, \mathrm{Ba}, \mathrm{Si}$ e Sr, exceto Co. As leituras das concentrações foram realizadas através da espectrometria de emissão atômica com plasma de argônio. Na matéria seca, os elementos que apresentaram maior destaque é a concentração são $\mathrm{Mg}$ e Mn. As maiores concentrações na infusão da erva-mate, em ordem decrescente, foram: $K$; $\mathrm{Mg}$; $\mathrm{S}$; Ca e P. Quanto à solubilidade do elemento, em relação a sua concentração na matéria seca foi a seguinte: $\mathrm{B}>\mathrm{S}_{-} \mathrm{SO}_{4}>\mathrm{Zn}$ $>\mathrm{K}>\mathrm{P}>\mathrm{Mg}=\mathrm{Mn}>\mathrm{Cu}>\mathrm{Cr}$, os demais apresentaram valores menores que $30 \%$. A infusão da erva-mate apresenta altas concentrações de $K, \mathrm{Mg}$ e Mn (por ser um micronutriente), intermediárias de $S$, Ca e P, baixa de Al e zero de $C d$ e $P b$.

Palavras-chave: composição mineral, erva-mate, infusão, Ilex paraguariensis.
SUMMARY

The mineral composition of three commercial mate products and of the infusions thereof were evaluated, being four replicates used. In the industrial processing of 'chimarrão' mateherb, leaves and twigs are employed, having an approximate proportion of $70 \%$ and $30 \%$ respectively. Both in the commercial mate products and their infusions $N$ was determined by microKjeldahl whereas $\mathrm{P}, \mathrm{K}, \mathrm{Ca}, \mathrm{Mg}, \mathrm{S}, \mathrm{B}, \mathrm{Cu}, \mathrm{Fe}, \mathrm{Mn}, \mathrm{Ni}, \mathrm{Zn}, \mathrm{Al}, \mathrm{Cd}$, $\mathrm{Co}, \mathrm{Cr}, \mathrm{Na}$, and $\mathrm{Pb}$ were analysed either by atomic absorption or by argon plasma. In the commercial product $\mathrm{Mg}$ and Mn were the main elements. In the infusions higher concentrations were in decreasing order: $K, M g, S, C a$ and $P$. Solubility obeyed the order: $\mathrm{B}>\mathrm{S}-\mathrm{SO}_{4}>\mathrm{Zn}>\mathrm{K}>\mathrm{P}>\mathrm{Mg}=\mathrm{Mn}>\mathrm{Cu}>\mathrm{Cr}$. The remaining elements showed lower $30 \%$ soluble proportions. The infusion has high concentrations of $\mathrm{K}, \mathrm{Mg}$ and $\mathrm{Mn}$ ( $\mathrm{a}$ micronutrient), intermediate ones for $S, C a$ and $P$, low level of $A l$, and zero concentrations of $\mathrm{Cd}$ and $\mathrm{Pb}$.

Key words: Mineral composition, mate-herb, infusion, Ilex paraguariensis.

\section{INTRODUÇÃO}

A planta de erva-mate (Ilex paraguariensis St. Hil.), espécie nativa da América do Sul é comum na vegetação nativa de uma extensa área que abrange Brasil, Argentina e Paraguai. Na região sul do Brasil, a cultura desempenha um importante papel sócio-econômico e ambiental, principalmente nas pequenas propriedades agrícolas (RODIGHERI et al., 1996).

A produção brasileira anual está em torno de 206.000t, sendo os principais estados, em ordem

\footnotetext{
${ }^{1}$ Engenheiro Agronomo, MS., Doutorando do Centro de Energia Nuclear na Agricultura (CENA), Universidade de São Paulo (USP), Laboratório de Nutrição Mineral de Plantas, CP 96, 13400-970, Piracicaba, SP. Bolsista da FAPESP. E-mail: regesh@cena.usp.br Autor para correspondência.

${ }^{2}$ Engenheiro Agrônomo, Professor Catedrático do Centro de Energia Nuclear na Agricultura (CENA/USP), CP 96, 13400-970 Piracicaba, SP. Bolsista do CNPq. E-mail: mala@ cena.usp.br.
} 
decrescente, Paraná, Santa Catarina, Rio Grande do Sul e Mato Grosso do Sul (IBGE, 1998). O maior consumidor é o Estado do RS, representando cerca de $50-60 \%$ da comercialização no país, no entanto, essa cultura muito vem se expandindo, chegando a estados do norte com consumo significativo (TORMEN, 1995). Atualmente, atingindo o mercado europeu, onde é utilizado como infusão (SANZ \& ISASA, 1991).

O fato de a erva-mate ser uma cultura que tem suas folhas e ramos finos retirados regularmente, implica a exportação maciça de nutrientes, ficando a reposição nutricional necessária para manter a produtividade (GAIAD \& LOPES, 1986). No entanto, além dos estudos de nutrição da planta, também se torna importante o conhecimento dos elementos químicos que são consumidos pelo homem por meio da ingestão da bebida.

A erva-mate, consumida como chá ou chimarrão, pode ser muito mais que uma bebida agradável e estimulante. Segundo STAGG \& MILLIN (1975), a ingestão da infusão pode ser uma importante fonte de minerais essenciais e vitaminas. Provavelmente essa planta apresente propriedades terapêuticas, prevenindo cáries dentárias e profilaxia e tratamento de doenças coronárias, vasculares e inclusive arteriosclerose. Os autores ainda ressaltam que, infelizmente, pouco se estuda sua potencialidade, pois os principais países envolvidos com o seu consumo são poucos Entre esses estão a Rússia e Índia.

O grande consumo e os poucos estudos sobre a composição mineral após a industrialização da planta fundamentaram a necessidade da realização do presente trabalho, com o objetivo de quantificar e avaliar a concentração mineral no produto comercial da erva-mate para chimarrão.

\section{MATERIAIS E MÉTODOS}

Selecionaram-se três amostras comerciais de erva-mate tipo chimarrão, de ampla aceitação pelo consumidor para determinação da concentração mineral na matéria seca e na infusão. $\mathrm{Na}$ industrialização da erva-mate, são utilizadas folhas, pecíolos e ramos finos, tendo uma composição aproximada de $30 \%$ ramos e $70 \%$ folhas, que são beneficiados para posterior comercialização. O preparo das amostras e as análises foram efetuadas no Laboratório de Nutrição Mineral de Plantas do Centro de Energia Nuclear na Agricultura (CENA/USP).

Para a determinação da composição mineral da matéria seca, as amostras foram moídas em moinho "tipo Willey" (com 3 facas, 1725rpm, malha 20 mesh, moído por 3 minutos) e digeridas. A con- centração de $\mathrm{N}$ total foi determinada via digestão sulfúrica e posterior destilação em destilador de arraste de vapor semi - micro Kjeldhal, enquanto os demais elementos: $\mathrm{P}, \mathrm{K}, \mathrm{Ca}, \mathrm{Mg}, \mathrm{S}, \mathrm{B}, \mathrm{Cu}, \mathrm{Fe}, \mathrm{Mn}$, $\mathrm{Ni}, \mathrm{Zn}, \mathrm{Al}, \mathrm{Cd}, \mathrm{Co}, \mathrm{Cr}, \mathrm{Na}$, e $\mathrm{Pb}$ foram determinados via digestão nitro perclórica e posterior leitura em espectrometria de emissão atômica com plasma de argônio. Para determinação dos elementos químicos na infusão, utilizaram-se $70 \mathrm{~g}$ de erva-mate para um litro de água desionizada a $80^{\circ} \mathrm{C}$, simulando-se a temperatura para chimarrão, mantendo-se em contato até esfriar, sendo posteriormente coada. Os elementos analisados na infusão foram os determinados na matéria seca e, além destes $\mathrm{Mo}, \mathrm{Ba}, \mathrm{Si}, \mathrm{Sr}$, exceto Co. A concentração de $\mathrm{N}$ total foi determinada com a destilação de $20 \mathrm{~m} \ell$ da infusão e com leitura em espectrometria de emissão atômica com plasma de argônio para os demais elementos (MALAVOLTA et al., 1997).

A fração solúvel, em água quente, dos elementos contidos na massa seca foi calculada a partir da seguinte fórmula:

$\mathrm{FS}=(\mathrm{CI} \times 100) /(\mathrm{CMS} \times 70 / 1000)$

onde: FS = fração solúvel (\%);

$\mathrm{CI}=$ concentração média do elemento na infusão $\left(\mathrm{mg} \ell^{-1}\right)$;

CMS = concentração média do elemento na matéria seca $\left(\mathrm{mg} \mathrm{kg}^{-1}\right)$.

Foi utilizado um delineamento experimental inteiramente casualizado, com quatro unidades experimental por amostra. Os resultados da concentração de elementos foram interpretados por meio da análise da variância (SAS, 1988).

\section{RESULTADOS E DISCUSSÃO}

\section{Matéria seca}

Na tabela 1, estão apresentados os teores dos elementos encontrados na matéria seca da ervamate. Os resultados de $\mathrm{K}, \mathrm{Ca}, \mathrm{Mg}, \mathrm{Cu}$ e $\mathrm{Mn}$ foram semelhantes aos encontrados por SANZ \& ISASA (1991), enquanto o $\mathrm{Fe}$ foi inferior e o $\mathrm{Na}$ superior.

Comparando os resultados com os teores encontrados nas folhas usadas para diagnose do café e cacau, ambos plantas estimulantes, verifica-se que o Mg apresenta teores semelhantes nas três culturas, evidenciando que a erva-mate apresenta alta concentração do elemento, pois o material utilizado em análise foi a erva-mate comercial, ou seja, aproximadamente $30 \%$ de ramos e $70 \%$ de folhas. Tais resultados corroboram os de VALDUGA $\boldsymbol{e t} \boldsymbol{a l}$. (1997). Os autores, ao analisarem folhas de ervamate do município de Paulo Freitas - PR, encontra- 
Tabela 1 - Teores de elementos minerais na matéria seca da erva-mate tipo chimarrão $^{(1)}$, café $^{(2)}$ e cacau $^{(2)}$. Piracicaba, 1999.

\begin{tabular}{|c|c|c|c|c|c|c|}
\hline Elementos & mínimo & máximo & média & $\mathrm{CV}^{(3)}$ & café & cacau \\
\hline & \multicolumn{3}{|c|}{ 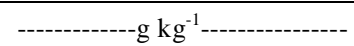 } & $(\%)$ & \multicolumn{2}{|c|}{ 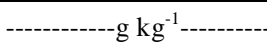 } \\
\hline $\mathrm{N}$ & 15 & 17 & 16 & 3 & $29-32$ & $19-23$ \\
\hline $\mathrm{P}$ & 0,8 & 1,2 & 0,9 & 3 & $1,6-1,9$ & $1,5-1,8$ \\
\hline K & 12 & 14 & 13 & 2 & $22-25$ & $17-20$ \\
\hline $\mathrm{Ca}$ & 6,0 & 6,6 & 6,3 & 2 & $13-15$ & $9-12$ \\
\hline $\mathrm{Mg}$ & 4,3 & 5,2 & 4,9 & 2 & $4,0-4,5$ & $4-7$ \\
\hline \multirow[t]{2}{*}{$\mathrm{S}$} & 0,8 & 1,1 & 0,9 & 6 & $1,5-2,0$ & $1,7-2,0$ \\
\hline & \multicolumn{3}{|c|}{-------------mg kg-1 ------------- } & & \multicolumn{2}{|c|}{---------mg kg ${ }^{-1}$-------- } \\
\hline B & 26 & 37 & 32 & 6 & $50-60$ & $30-40$ \\
\hline $\mathrm{Cu}$ & 7,6 & 10,7 & 8,9 & 2 & $11-14$ & $10-15$ \\
\hline $\mathrm{Fe}$ & 103 & 286 & 185 & 15 & $100-130$ & $150-200$ \\
\hline $\mathrm{Mn}$ & 655 & 1050 & 880 & 3 & $80-100$ & $150-200$ \\
\hline $\mathrm{Ni}$ & 0,01 & 3,5 & 1,9 & 14 & - & - \\
\hline $\mathrm{Zn}$ & 38 & 43 & 40 & 3 & $15-20$ & $50-70$ \\
\hline $\mathrm{Al}$ & 257 & 616 & 403 & 8 & - & - \\
\hline $\mathrm{Cd}$ & - & - & $<0,01$ & - & - & - \\
\hline $\mathrm{Co}$ & - & - & $<0,01$ & - & - & - \\
\hline $\mathrm{Cr}$ & 1,0 & 2,2 & 1,5 & 18 & - & - \\
\hline $\mathrm{Na}$ & 16 & 87 & 39 & 57 & - & - \\
\hline $\mathrm{Pb}$ & - & - & $<0,03$ & - & - & - \\
\hline
\end{tabular}

(1) Média de quatro repetições e três amostras comerciais com aproximadamente $30 \%$ de ramos e $70 \%$ de folhas;

(2) Teores na folha usada para diagnose considerados adequados segundo MALAVOLTA et al. (1997);

${ }^{(3)}$ Coeficiente de variação.

ram teores de $9,1 \mathrm{~g} \mathrm{~kg}^{-1}$ de $\mathrm{Mg}$. Entre os micronutrientes, o teor de Fe é semelhante ao encontrado nas folhas de cacau, enquanto o Mn apresentou uma concentração muito superior na erva-mate, o que também fora observado por VALDUGA et al. (1997). Os demais nutrientes apresentaram menor concentração que no café e cacau.

\section{Infusão}

Por várias funções metabólicas, muitos elementos presentes na infusão da erva-mate são essenciais à saúde humana. Portanto, o consumo regular de chá pode contribuir para suprir as necessidades destes elementos. A maioria dos minerais presentes no chá da erva-matesão aqueles encontrados em outras plantas e também alguns alimentos vegetais. Assim, é improvável que esses minerais tragam perigo para a saúde, particularmente na infusão, processo no qual, diferente da alimentação, o fornecimento de elementos é indireto, ou seja, somente a fração solúvel na infusão é consumida (NATESAN \& RANGANATHAN, 1990).

Os valores nutricional e terapêutico do chá são descritos por STAGG \& MILLIN (1975). Os autores relatam que, com a quantidade da infusão consumida, a concentração do elemento e a porcentagem solúvel, é possível estimar a quantidade do elemento consumido por uma população.

A erva-mate apresenta uma alta concentração de K na infusão (Tabela 2), resultado que corrobora os encontrados por RAMALLO et al. (1998). Segundo STAGG \& MILLIN (1975), o K é importante para pessoas hipertensas. Por sua vez, o Mn exerce funções no organismo através da ativação de enzimas (cofator) ou por ser parte integrante de sistemas enzimáticos (ROSA, 1988), a erva-mate se torna uma importante fonte do elemento, pois a maioria dos alimentos apresentam concentração muito pequenas (STAGG \& MILLIN, 1975). De acordo com NATESAN \& RANGANATHAN (1990), o chá tem uma importância reconhecida como fornecedor de Co. Os autores afirmam que o elemento reduz doenças cardiovasculares. Entretanto, sua presença não foi constatada na matéria seca da erva-mate (Tabela 1) e conseqüentemente esse elemento não foi analisado na infusão. $\mathrm{O} \mathrm{Cr}$ é essencial para funcionamento do fator tolerante da glucose. Embora pouco ainda se saiba sobre alguns elementos como $\mathrm{Cr}, \mathrm{Ni}$ e $\mathrm{Zn}$, é reconhecida a essencialidade deles.

Não foram detectados teores de $\mathrm{Pb}$ e $\mathrm{Cd}$ na infusão de erva mate. De acordo com TSUSHIDA \& TAKEO (1976), o Pb pode variar de acordo com a proximidade de áreas metropolitanas, mas o mesmo não foi constatado com o $\mathrm{Cd}$. Por outro lado, MICHIE \& DIXON (1977) revelam que as folhas de plantas usadas para preparo de infusões apresentam a capacidade de complexar metais que beneficia a retenção de metais tóxicos como o $\mathrm{Pb}$ e $\mathrm{Cd}$, evitando sua ingestão.

As maiores concentrações na infusão da erva-mate, em ordem decrescente, foram: $\mathrm{K} ; \mathrm{Mg} ; \mathrm{S}$; $\mathrm{Ca}$ e $\mathrm{P}$. Quanto à solubilidade dos elementos, em relação a sua concentração na matéria seca foi a seguinte: $\mathrm{B}>\mathrm{S}_{-} \mathrm{SO}_{4}>\mathrm{Zn}>\mathrm{K}>\mathrm{P}>\mathrm{Mg}=\mathrm{Mn}>\mathrm{Cu}$ $>\mathrm{Cr}$, os demais apresentaram valores menores que $30 \%$ (Tabela 2). O Ni e Na apresentaram solubilidade maior que $100 \%$, o que provavelmente, é erro experimental relacionado à grande diferença entre as concentrações mínima e máxima, bem como pelo baixo valor absoluto. Na análise da variância, podese observar que alguns elementos, como Na na matéria seca (Tabela 1) e $\mathrm{Ni}, \mathrm{Sr}, \mathrm{Cu}, \mathrm{Cr}$, e Ca na infusão (Tabela 2) apresentam alto coeficiente de variação. Isso pode ser atribuído ao fato de a erva-mate ser um 
Tabela 2 - Teores de elementos minerais na infusão da erva-mate tipo chimarrão e porcentagem média solúvel em relação à concentração na matéria seca $^{(1)}$. Piracicaba, 1999

\begin{tabular}{lccccc}
\hline Elemento & mínimo & máximo & média & $\mathrm{CV}^{(2)}$ & $\begin{array}{c}\text { Fração } \\
\text { solúvel }\end{array}$ \\
& & & & & $(\%)$ \\
& & & & & \\
$\mathrm{N}$ & 9,1 & 12,2 & 11 & 14 & 1 \\
$\mathrm{P}$ & 32 & 49 & 41 & 20 & 65 \\
$\mathrm{~K}$ & 619 & 735 & 683 & 9 & 75 \\
$\mathrm{Ca}$ & 31 & 58 & 44 & 30 & 10 \\
$\mathrm{Mg}$ & 168 & 200 & 188 & 9 & 55 \\
$\mathrm{~S}-\mathrm{SO}_{4}$ & 51 & 69 & 58 & 17 & 92 \\
$\mathrm{~B}$ & 2,02 & 2,38 & 2,22 & 8 & 99 \\
$\mathrm{Cu}$ & 0,16 & 0,44 & 0,28 & 49 & 45 \\
$\mathrm{Fe}$ & 0,31 & 0,35 & 0,33 & 6 & 2,5 \\
$\mathrm{Mn}$ & 31 & 37 & 34 & 8 & 55 \\
$\mathrm{Mo}$ & 0,02 & 0,04 & 0,03 & 18 & - \\
$\mathrm{Ni}$ & 0,05 & 0,31 & 0,20 & 64 & 150 \\
$\mathrm{Zn}$ & 2,14 & 2,38 & 2,25 & 5 & 80 \\
$\mathrm{Al}$ & 2,99 & 3,75 & 3,43 & 11 & 12 \\
$\mathrm{Ba}$ & 1,15 & 1,34 & 1,25 & 8 & - \\
$\mathrm{Cd}$ & 0,00 & 0,00 & 0,00 & - & - \\
$\mathrm{Cr}$ & 0,03 & 0,06 & 0,04 & 39 & 38 \\
$\mathrm{Na}$ & 2,85 & 3,54 & 3,23 & 10 & 118 \\
$\mathrm{~Pb}$ & 0,00 & 0,00 & 0,00 & - & - \\
$\mathrm{Si}$ & 5,22 & 7,47 & 6,31 & 18 & - \\
$\mathrm{Sr}$ & 0,36 & 0,81 & 0,52 & 50 & - \\
& & & & & \\
\hline & & & & &
\end{tabular}

(1) Solução preparada com $70 \mathrm{~g}$ de massa de matéria seca (aproximadamente $30 \%$ de ramos e $70 \%$ de folhas) para $1 \ell$ de água. ${ }^{(2)}$ Coeficiente de variação.

produto comercial e haver pequenas variações na relação ramos-folhas das amostras.

Estudos sobre Al relatam que altas concentrações ocorrem em regiões nucleares do tecido cerebral no hipocampo de pacientes com doença de Alzheimer. No entanto, não se sabe se o $\mathrm{Al}^{3+}$ está relacionado etiologicamente com a doença ou se tem alguma afinidade com essas regiões com anomalias (MACDONALD $\boldsymbol{e t}$ al., 1987). Baseado na toxidade do $\mathrm{Al}$ e seus efeitos no crescimento, reprodução, tecido ósseo e físiologia cerebral, NATESAN \& RANGANATHAN $\boldsymbol{e}$ t $\boldsymbol{a l}$. (1990) citam que Candriam concluiu que o consumo de até 20mg Al dia ${ }^{-1}$ é inofensivo. A partir destes resultados, pode-se constatar que a ingestão de $\mathrm{Al}$ por meio da erva mate é muito pequena (Tabela 2).

O presente estudo apresenta as concentrações dos elementos minerais que se encontram na erva mate, mas não se pode assumir que estão totalmente biodisponíveis. Há inúmeros compostos orgânicos, especialmente polifenois, cuja forma química ainda não é conhecida. Provavelmente, vários elementos absorvidos pelo trato digestivo dependem destas formas químicas. Estudos futuros são necessários para se obterem informações sobre a biodisponibilidade, potencial terapêutico e nutricional destes elementos consumidos na forma de infusões, uma vez que a erva mate pode ter uma importante contribuição para saúde humana.

\section{CONCLUSÕES}

$\mathrm{Na}$ matéria seca, os elementos que apresentam maior destaque são as concentrações de Mg e Mn.

A infusão da erva-mate apresenta altas concentrações de $\mathrm{K}, \mathrm{Mg}$ e $\mathrm{Mn}$, intermediárias de $\mathrm{S}$, $\mathrm{Ca}$ e $\mathrm{P}$, baixo de $\mathrm{Al}$ e zero de $\mathrm{Cd}$ e $\mathrm{Pb}$.

\section{REFERÊNCIAS BIBLIOGRÁFICA}

GAIAD, S., LOPES, E.S. Ocorrência de micorriza vesiculararbuscular em erva-mate (Ilex paraguariensis St. Hil.). Boletim de Pesquisa Florestal, Curitiba, v.12, p.21-29, 1986.

IBGE. Anuário Estatístico do Brasil. Rio de Janeiro, v.58, p.336, 1998

MACDONALD, T.L., HUMPHREYS, W.G., MARTIN, R.B. Promotion of tubulin assembly by aluminium ion in vitro. Science, Washington, v.236, p.183-186, 1987.

MALAVOLTA, E., VITTI, G.C., OLIVEIRA, S.A. Avaliação do estado nutricional das plantas: princípios e aplicações. $2^{a}$ ed. Piracicaba: Associação Brasileira para Pesquisa da Potassa e do Fosfato, 1997. 319p.

MICHIE, N.D., DIXON, E.J. Distribution of lead and other metals in tea leaves, dust and liquors. Journal Science Food Agriculture, London, v.28, p.215-224, 1977.

NATESAN, S., RANGANATHAN, V. Content of various elements in different parts of the tea plant and in infusions of black tea from southern India. Journal Science Food Agriculture, London, v.51, p.125 - 139, 1990.

RAMALLO, L.A., SMORCEWSKI, M., VALDEZ, E.C. et al. Contenido nutricional del extracto acuoso de la yerba mate en tres formas diferentes de consumo. La Alimentación Latinoamericana, Bueno Aires, n.225, 1998.

RODIGHERI, H.R., SCHLOSSNACHER NETO, L., CICHACZEWSKI, I.F. Custos, produtividade e renda da erva-mate cultivada na região de Guarapuava, PR. Colombo : EMRAPA-CNPF, 1996. 22p. (Circular Técnica, 24).

ROSA, I.V. Micronutrientes no animal: funções no metabolismo e consequiências de carências e excessos. In: FERREIRA, M.E., CRUZ M.C.P. (Ed.) Micronutrientes na agricultura. Piracicaba: POTAFOS/CNPq, 1991. p.35-64.

SANZ, M.D.T., ISASA, M.E.T. Elementos minerales en la yerba mate (Ilex paraguariensis St. H.). Archivos Latinoamericanos de Nutricion, Caracas, v.61, n.3, p.441-454, 1991. 
SAS INSTITUTE INC. SAS procedures guide. Release 6.03. Cary, NC : SAS Inst, 1988. 441p.

STAGG G.V., MILLIN, D.J. The nutritional and therapeutic value of tea - a review. Journal Science Food Agriculture, London, v.26, p.1439-1459, 1975.

TORMEN, M.J. Economia ervateira brasileira. In: WINGE, H., FRREIRA, A.G., MARIATH, J.E.A., et al. Erva-mate: biologia e cultura no Cone Sul. Porto Alegre : Universidade/UFRGS, 1995. p.27-40.
TSUSHIDA, T., TAKEO, T. Zinc, copper, lead and cadmium content in green tea. Journal Science Food Agriculture, London, v.28, p.255-258, 1977.

VALDUGA, E., FREITAS, R.J.S.de, REISMANN, C.B., et $\boldsymbol{a}$ l. Caracterização química da folha de Ilex paraguariensis St. Hil. (erva-mate) e de outras espécies utilizadas na adulteração do mate. Boletim do Centro de Pesquisa e Processamento de Alimentos, Curitiba, v.15, n.1, p.25-36, 1997.

Ciência Rural, v. 31, n. 5, 2001. 